\title{
LES DIFFERENTES FORMES DE VIOLENCES EN MILIEU ORGANISATIONNEL
}

\begin{abstract}
Eva EIN-ELI ${ }^{1}$
Résumé: Cette étude a pour objectif de comparer l'importance des diverses violences (morales, sexuelles, physiques et environnementales) en milieu organisationnel et de repérer l'impact de variables sociodémographiques et organisationnelles sur chacun d'elles. Un questionnaire a été proposé sur un échantillon de 406 travailleurs français. L'analyse des résultats fait apparaitre 4 classes de violences: l'atteinte à la dignité, l'atteinte à l'intégrité de la personne, le dénigrement de la personne et le dénigrement du travail. Notre travail complète et affine les résultats d'études antérieures. Il est en cohérence avec les résultats de la recherche de Leymann portant sur le mobbying, ce qui lui confère une certaine validité.
\end{abstract}

Mots clé: violences, morale, sexuelle, physique et environnementale, organisation.

\section{Introduction}

Le recensement de la littérature témoigne du peu de travaux portant sur les violences au travail. On peut citer les recherches de Jauvin, Dompierre et Vezina (1999) qui classifient les violences en fonction de la nature des comportements qu'elles soutendent. Ces auteurs différencient les violences physiques (agressions, attaques, séquestration), sexuelles (harcèlement sexuel, attouchements et viol) et morales (harcèlement moral et discrimination) auxquelles Ein-Eli (2018) ajoute les violences environnementales.

La plupart des chercheurs se centrent sur un type de violences. Ainsi, Hirigoyen (2017) s'intéresse au harcèlement moral au travail, Cox (2012) au harcèlement psychologique au travail, Desrumaux (2007) travaille sur le harcèlement moral et les comportements de survictimation. Nous parvenons au même constat lorsque nous consultons les rapports et travaux institutionnels. La DARES prend en compte les comportements hostiles au travail, l'IFOP se penche sur le sexisme et le harcèlement sexuel et le défenseur des droits s'appuie sur le baromètre des perceptions au travail pour étudier les discriminations au travail.

Rares sont les recherches comparant ces violences. On peut néanmoins citer la recherche menée par Paoli et Merllié (2000) portant sur les conditions de travail en

\footnotetext{
1 LAPPS, Université Paris Nanterre, eva.ee@parisnanterre.fr
} 
Europe et la troisième enquête européenne sur les conditions de travail (2000). Paoli et Merllié (2000) montrent que la violence morale au travail, illustrée ici par l'intimidation et le harcèlement moral, est plus fréquente que la violence physique ou le harcèlement sexuel. L'enquête européenne de 2000 compare les différentes formes de harcèlement dans le milieu organisationnel européen mais elle ne compare pas toutes les formes de violences en milieu organisationnel ou leurs prévalences les unes par rapport aux autres. Une étude menée au Portugal par Antunes, Biscaia, Conceição, Fronteira, Craveiro, Flores, Santos et Ferrinho (2002) qui a évalué l'exposition des participants à diverses violences au travail: violence morale (harcèlement verbal, pression morale, discrimination), violence physique et sexuelle (harcèlement sexuel) dans un hôpital $(n=270)$ et dans un centre de soins $(n=221)$. Elle révèle la place prépondérante du harcèlement verbal et des brimades.

En 2007, Tragno, Duveau et Tarquinio analysent 28 articles portant sur les violences physiques et sur les agressions physiques au travail et soulignent des différences dans les définitions de ces violences physiques ou agressions, les outils et méthodes ainsi que les causes et conséquences retenues comme sources de violence, ce qui empêche toute comparaison.

Notre recherche se propose de:

- Comparer le poids respectif des violences (physiques, sexuelles, morales et environnementales) en milieu organisationnel;

- Repérer l'impact des variables sociodémographiques et organisationnelles sur chacun de ces types de violences au travail.

\section{Méthode}

L'échantillon est composé de 406 travailleurs, âgés de 24 ans à 64 ans ( $M=40,08$ ans; $\mathrm{ET}=2,5$ ans) et dont $74,46 \%$ vivent en couple. L'ancienneté dans l'organisation varie de 1 à 43 ans ( $M=9$ ans et 8 mois; $E T=8$ ans et 5 mois). $78 \%$ des participants ont des contrats à long terme ( $C D I$ ou Titulaire de la fonction publique), $9 \%$ des contrats à périodes déterminés (CDD, vacataire, saisonnier) et $1 \%$ ont un statut libéral ou autoentrepreneur. Enfin $48,28 \%$ des participants ont un contact avec le public ou la clientèle dans le cadre de leur travail (par exemple, infirmiers, commerciaux, vendeurs, enseignants, policiers).

Le questionnaire a été construit en retenant des items issus de la littérature scientifique (Desrumaux, 2011; Zid, Haines, Jeoffrion \& Barre, 2016), de rapports gouvernementaux (IFOP, DARES, Baromètre du défenseur des droits sur les discriminations), d'articles de lois (article L 1153-1 du Code du travail) et d'arrêts jurisprudentiels (arrêt du 7 Février 2017 de la cour d'appel d'Orléans).

Il a ensuite été soumis à:

(a) un comité d'experts constitué par trois enseignants-chercheurs (un en droit et deux en psychologie du travail) afin que soit testée sa validité de contenu.

(b) dix salariés du secteur privé et public afin que soit vérifiée la clarté de chaque item. Ainsi chaque participant a évalué sur une échelle allant de (1) pas du tout à (5) tout à fait la clarté de chaque item. 
Le questionnaire définitif comprenant 16 items (voir annexe) a été diffusé en ligne via un réseau professionnel et personnel (Linkedin, Viadéo, Facebook) et sur un site internet sans lien avec le travail.

Tous les participants ont répondu volontairement de manière individuelle. La passation a duré environ 15 minutes.

\section{Résultats}

Nous présenterons (3.1) les résultats descriptifs globaux, puis (3.2) par classe de violences.

\subsection{Résultats globaux}

93\% des participants ont au moins subi une fois une violence au travail. Autrement dit, seuls $7 \%$ des participants disent ne pas avoir personnellement dû faire face à de la violence au travail au cours des six derniers mois.

Notons que $4 \%$ des participants qui ont subi des violences au cours des six derniers mois considèrent que cela ne les a pas gênés.

Tableau 1

Violences au travail subies et considérées comme gênantes classées par type

\begin{tabular}{|c|c|c|c|c|c|c|c|c|c|c|c|c|c|c|c|c|}
\hline & \multicolumn{4}{|c|}{$\begin{array}{l}\text { Violence physique } \\
\text { (5 items)* }\end{array}$} & \multicolumn{4}{|c|}{$\begin{array}{l}\text { Violence sexuelle } \\
\quad(4 \text { items)** }\end{array}$} & \multicolumn{4}{|c|}{$\begin{array}{l}\text { Violence morale } \\
\text { (4 items) }\end{array}$} & \multicolumn{4}{|c|}{$\begin{array}{c}\text { Violence } \\
\text { environnementale } \\
(3 \text { items })^{* * *}\end{array}$} \\
\hline & \multicolumn{2}{|c|}{$\begin{array}{l}\text { Réponse } \\
\text { positive }\end{array}$} & \multicolumn{2}{|c|}{$\begin{array}{l}\text { Ça me } \\
\text { gêne }\end{array}$} & \multicolumn{2}{|c|}{$\begin{array}{l}\text { Réponse } \\
\text { positive }\end{array}$} & \multicolumn{2}{|c|}{$\begin{array}{l}\text { Ça me } \\
\text { gêne }\end{array}$} & \multicolumn{2}{|c|}{$\begin{array}{l}\text { Réponse } \\
\text { positive }\end{array}$} & \multicolumn{2}{|c|}{$\begin{array}{l}\text { Ça me } \\
\text { gêne }\end{array}$} & \multicolumn{2}{|c|}{$\begin{array}{l}\text { Réponse } \\
\text { positive }\end{array}$} & \multicolumn{2}{|c|}{$\begin{array}{l}\text { Ça me } \\
\text { gêne }\end{array}$} \\
\hline & $\mathrm{N}$ & $\%$ & $\mathrm{~N}$ & $\%$ & $\mathrm{~N}$ & $\%$ & $\mathrm{~N}$ & $\%$ & $\mathrm{~N}$ & $\%$ & $\mathrm{~N}$ & $\%$ & $\mathrm{~N}$ & $\%$ & $\mathrm{~N}$ & $\%$ \\
\hline $\begin{array}{l}\text { Au moins } \\
1 \text { item }\end{array}$ & 115 & $28 \%$ & 115 & $28 \%$ & 225 & $56 \%$ & 194 & $48 \%$ & 332 & $82 \%$ & 317 & $78 \%$ & 306 & $75 \%$ & 301 & $74 \%$ \\
\hline $\begin{array}{l}\text { Tous les } \\
\text { items }\end{array}$ & 1 & $0 \%$ & 6 & $1 \%$ & 18 & $4 \%$ & 13 & $3 \%$ & 62 & $15 \%$ & 44 & $11 \%$ & 0 & $0 \%$ & 0 & $0 \%$ \\
\hline
\end{tabular}

* impact de l'âge des participants

** impact du statut marital et de la profession (contact vs non contact avec le public)

*** impact du nombre d'enfants

Les pourcentages de violences subies sont significativement différents selon les types de violences $\left(\mathrm{chi}^{2}=293.49, \mathrm{p}<.01\right)$.

Ainsi, plus des $3 / 4$ des participants déclarent avoir subi de la violence morale (82\%) ou de la violence environnementale (75.4\%) au travail. Seuls $28 \%$ font référence à de la violence physique $(p<.05)$.

$56 \%$ signalent avoir subi de la violence sexuelle. Parmi ceux-ci $4 \%$ ont coché les 4 items concernant ce type de violence. Pourtant $8 \%$ des participants déclarant avoir subi des violences sexuelles affirment que cela ne les ont pas gênés. 
Notons que $15 \%$ des participants disent avoir subi toutes les formes de violences morales que nous avons répertoriées dans le questionnaire.

Par ailleurs, on constate que plus le participant est jeune, plus il est susceptible de subir des violences physiques et d'en être gêné $(r=.14, p<.05)$. Plus il a d'enfants, plus il est susceptible de subir des violences environnementales $(r=.14, p<.05)$ et d'en être gêné $(r=.14, p<.05)$. Enfin les participants qui vivent seuls ou qui ont des contacts avec le public dans le cadre de leur travail subissent plus fréquemment des violences sexuelles et en sont gênés (respectivement $r=.14$ et $r=.21, p<.05$ ).

\subsection{Résultats par classe de violences}

L'analyse des résultats par classe de violences fait apparaitre 4 classes de violences regroupées dans le tableau ci-dessous.

Les quatre classes de violences

Tableau 2

\begin{tabular}{|l|l|l|l|l|}
\hline \multicolumn{1}{|c|}{ Classes de violences } & \multicolumn{1}{|c|}{$\begin{array}{c}\mathbf{N}^{\circ} \text { des } \\
\text { Items }\end{array}$} & $\begin{array}{c}\text { \% de personnes } \\
\text { ayant subi des } \\
\text { violences }\end{array}$ & $\begin{array}{c}\text { \% de personnes } \\
\text { gênées par les } \\
\text { violences subies }\end{array}$ & Types de violences \\
\hline $\begin{array}{l}\text { Atteinte à l'intégrité de } \\
\text { la personne* }\end{array}$ & $\begin{array}{l}7,11,12, \\
16\end{array}$ & $6.75 \%$ & $5.75 \%$ & $\begin{array}{l}\text { Environnementale } \\
\text { Physique }\end{array}$ \\
\hline Atteinte à la dignité** & $3,5,13$ & $12.67 \%$ & $12 \%$ & $\begin{array}{l}\text { Physique } \\
\text { Sexuelle }\end{array}$ \\
\hline $\begin{array}{l}\text { Dénigrement de la } \\
\text { personne** }\end{array}$ & $\begin{array}{l}4,8,10, \\
14\end{array}$ & $32.25 \%$ & $26 \%$ & $\begin{array}{l}\text { Sexuelle } \\
\text { Morale }\end{array}$ \\
\hline Dénigrement au travail & $2,6,1,9$ & $59.25 \%$ & $54.75 \%$ & $\begin{array}{l}\text { Environnementale } \\
\text { Morale }\end{array}$ \\
\hline
\end{tabular}

* impact de l'âge $\quad * *$ impact du statut marital $* * *$ impact du nombre d'enfants

La première classe que nous avons appelée Atteinte à l'intégrité de la personne renvoie à des violences physiques (par exemple, être bousculée), et environnementales (par exemple, des actes de vandalisme) touchant la personne dans sa privacité. Plus de $80 \%$ des personnes ayant subi ces violences en ont été gênées. Plus le participant est jeune, plus il est susceptible de subir des atteintes à son intégrité $(r=-.11, p<.05)$.

La deuxième classe appelée Atteinte à la dignité regroupe des violences physiques (par exemple, être retenue contre son gré), et sexuelles (par exemple, subir des propositions à caractère sexuel) limitant la liberté d'action de la personne et sont considérées comme très gênantes par les participants qui les subissent (de 81 à 100\%). Plus le participant est jeune, plus il est susceptible de subir des atteintes à sa dignité $(r=-.12, p<.05)$. Le fait de vivre seul corrèle également avec le fait de subir des atteintes à la dignité $(r=.11, p<.05)$.

La classe Dénigrement de la personne comprend des violences sexuelles liées à l'apparence (par exemple, des remarques sur la tenue vestimentaire), et morales concernant leur activité professionnelle (par exemple, des critiques répétées du travail effectué). Moins le participant a d'enfants, plus il est susceptible de subir un dénigrement de sa personne $(r=-.12, p<.05)$ et plus cela le gênera $(r=.16, p<.01)$. 
La quatrième classe nommée Dénigrement au travail renvoie à des comportements de violences environnementales découlant d'un climat hostile, et de violences morales comme, par exemple, le fait d'être ignoré ou injustement critiqué au travail. Ces comportements sont considérés comme très gênants par les participants qui les subissent.

Une analyse des résultats par auteur des actes violents montre que les collègues manifestent davantage de violences portant atteinte à l'intégrité de la personne que les supérieurs $(p<.001)$. En revanche les supérieurs font preuve de davantage de violences portant atteinte à la dignité de la personne $(p<.05)$ ou dénigrant son travail $(p<.0001)$. Notons que les clients/usagers manifestent davantage de dénigrement, que ce soit envers la personne ou envers son travail $(p<.001)$.

\section{Discussion-conclusion}

Nous nous attendions à une moyenne de $25 \%$ de victimes conformément aux statistiques gouvernementales (rapport sur le harcèlement sexuel de l'IFOP, 2019). Nos résultats (au moins une violence en 6 mois en 2019 pour 93\% des participants) sont très largement supérieurs. Le fait que différents secteurs socio-professionnels et différents profils socio-démographiques soient représentés, semble indiquer que les violences au travail sont présentes dans le monde du travail dans sa globalité.

Ces résultats corroborent les études européennes puisque $82 \%$ des participants disent avoir subi au moins une des violences morales proposées dans le questionnaire et $78 \%$ s'en déclarent gênés. Quinze pour cent ont répondu positivement aux 4 items traduisant une violence morale. Ce chiffre révèle l'ampleur du phénomène de banalisation des violences.

$74 \%$ des participants ont déclaré avoir subi de la violence environnementale au cours des six derniers mois, ce qui place ce type de violences comme l'une des plus subies, juste avant la violence morale.

La violence sexuelle ne gêne que $48 \%$ des participants qui ont déclaré en avoir été victimes au moins une fois au cours des 6 derniers mois. Ainsi $8 \%$ des participants ne se sentent pas gênés par des attouchements ou des remarques sur leur tenue vestimentaire. Cela traduit-il une minimisation des faits? Leur connotation juridique et/ou les conséquences que cela engendrerait pour l'auteur de la violence poussentelles les victimes à se dire que ce n'est pas de la violence mais un comportement hostile au travail ? Si tel est le cas, où commence la violence sexuelle pour ces personnes ?

Cette minimisation de l'importance des actes à connotation sexuelle violents au travail rejoint la conclusion formulée par l'institut de sondage (IFOP) dans son enquête sur le harcèlement sexuel au travail (janvier 2014, note de synthèse page 3): cela " témoigne d'une certaine banalisation de ce type d'environnement au travail, si bien que cette situation semble être plus facilement tolérée, et ne pas entrer, par conséquent, pour la majorité des Français, dans le champ du harcèlement sexuel au travail ». On peut également se demander si la violence sexuelle au travail est perçue comme moins " grave " lorsqu'elle a lieu dans le cadre organisationnel. Dans ce cas, s'agit-il d'un ressenti, le lieu de travail étant considéré dans notre culture comme un lieu de 
souffrance (Dejours, 2016; Desrumaux, 2010; Hirigoyen, 2008) ou d'une méconnaissance de la loi ? Des entretiens semi directifs menés auprès de DRH du secteur privé et public vont dans le sens d'une méconnaissance de la loi et des règlements intérieurs (Ein-Eli, 2018).

En ce qui concerne les violences physiques, nous notons que $28 \%$ des participants en ont été victimes au cours des six derniers mois. Ce pourcentage est nettement supérieur à celui enregistré dans l'étude Danoise FTF, puisque 7\% des travailleurs interrogés se déclaraient victimes de violences physiques. Notons que tous déclarent avoir été gênés par ce type de violence subie. On peut probablement en déduire que la violence physique est considérée comme de la violence par les participants et n'est donc pas acceptable.

Cette recherche a permis de mettre en exergue 4 classes de violences en milieu organisationnel qui regroupent toutes les violences au travail. En cela, notre travail complète et affine les résultats des études portant sur un type de violences : physique (OIT, 2000), sexuelle (DARES, 2016; IFOP, 2019), morale (DARES, 2016; Einarsen \& Raknes, 1997) et environnementale (Desrumaux, 2011). II est en cohérence avec les résultats de la recherche de Leymann (1996) portant sur le mobbying, ce qui lui confère une certaine validité.

\section{References}

Antunes, A. R., Biscaia, A., Conceição, C., Fronteira, I., Craveiro, I., Flores, I., Santos, O., \& Ferrinho, P. (2002). Workplace violence in the health sector: Portuguese case studies, document préliminaire. Accessible à https://www.who.int/publications/ $\mathrm{m}$ /item/workplace-violence-in-the-health-sector---portuguese-case-studies

Cox, R. (2012). Harcèlement psychologique au travail: entre psychologisation et victimisation. Une étude de la jurisprudence arbitrale québécoise. Droit et société, 81, 343- 364.

Dejours, C. (2016). La méthodologie en psychopathologie du travail. Travailler, 35(1), 125-144.

Desrumaux, P. (2007). Harcèlement moral au travail, survictimation et problèmes du harceleur: quand les victimes sont jugées aussi responsables que leurs harceleurs. Les Cahiers Internationaux de Psychologie Sociale, 73(1), 61-73.

Desrumaux, P. (2010). Le travail, risque psychosocial ou facteur d'épanouissement : De la survie au bien-être. Le Journal des psychologies, Numéro spécial sur les risques psychosociaux, 283(10), 26-30.

Desrumaux, P. (2011). Le harcèlement moral au travail: réponses psychosociales, organisationnelles et cliniques. Rennes: Presses Universitaires de Rennes.

Ein-Eli, E. (2018). Le harcèlement sexuel au travail : la différence effective entre législation et pratique en entreprise. Communication affichée présentée au 59ème congrès de la Société Française de Psychologie-SFP, « psychologie, santé et société. De la théorie aux applications», Reims.

Einarsen, S., \& Raknes, B. I. (1997). Harassment in the workplace and the victimization of men. Violence and Victims, 12(3), 247-263. 
Hirigoyen, G. (2008). Biais comportementaux dans l'entreprise familiale: antécédents et impacts. La gouvernance de l'entreprise, 1901-1930.

Hirigoyen, M.-F. (2017). Le harcèlement moral au travail, 52ème édition. Paris: Que saisje.

Institut Français d'opinion publique-IFOP. (2019). Observatoire européen du sexisme et du harcèlement sexuel au travail. Accessible à https://www.ifop.com/publication/ observatoire-europeen-du-sexisme-et-du-harcelement-sexuel-au-travail/

Jauvin, N., Dompierre, J., \& Vezina, M. (1999). Recension documentaire sur la violence au travail. Accessible à http://www.santecom.qc.ca/BibliothequeVirtuelle/CLSC-CHSLDHaute-Ville-Des-Rivieres/RecensionViolenceTrav.pdf

Jauvin, N., Vézina, M., Bourbonnais, R., \& Dussault, J. (2006). Violence interpersonnelle en milieu de travail: une analyse du phénomène en milieu correctionnel québécois, Perspectives interdisciplinaires sur le travail et la santé, 8-1. Accessible à https://journals.openedition.org/pistes/3077?lang=en

Leymann, H. (1996). The content and development of mobbing at work. European Journal of Work and Organizational Psychology, 5(2), 165-184.

Paoli, P., \& Merllié, D. (2001). Third European Survey on Working Conditions 2000. Third European survey on working conditions 2000. European Foundation for the Improvement of Living and Working Conditions.

Tragno, M., Duveau, A., \& Tarquinio, C. (2007). Les violences et agressions physiques au travail: Analyse de la littérature. Revue Européenne de Psychologie Appliquée, 57(4), 237-255.

Zid, R., Haines, V., Jeoffrion, C., \& Barré, S. (2015). Liens entre le harcèlement moral et les émotions : I'effet modérateur des attributions causales. En V. Majer, P. Salengros, A. Di Fabio, \& C. Lemoine (eds.) Facteurs de la santé au travail: du bien être au mal être (pp. 113-124). L'Harmattan.

*** (2016). DARES analyses. Dans quels contextes les comportements sexistesau travail sont-ils le plus fréquent? Accessible à https://dares.travailemploi.gouv.fr/IMG/pdf/2016-046.pdf 


\section{Annexe: Questionnaire}

Ce questionnaire porte sur les violences au travail. II s'adresse à tous les travailleurs. Vous trouverez ci-dessous une série d'affirmations à caractère professionnel et personnel explorant votre ressenti et votre expérience pendant les six derniers mois.

Pour chaque affirmation vous répondrez aux modalités suivantes:

Réponse:

- o Oui

- o Non

Par qui?

- o Collègue

- o Supérieur

- o Client

- o Collectif de travail

- o Je ne sais pas

Cela me gêne?

- o Oui

- o Non

1. Au cours des 6 derniers mois, j'ai été victime de propos discriminants

2. Au cours des 6 derniers mois, j'ai subi un climat de travail hostile

3. J'ai été retenu(e) contre mon gré

4. J'ai subi des propos sexistes

5. J'ai été violenté(e)

6. J'ai été délibérément ignoré(e)

7. J'ai subi du vandalisme

8. J'ai subi des remarques sur mon physique ou ma tenue vestimentaire

9. Mon travail est injustement critiqué

10. J'ai entendu des propos à connotation sexuelle

11. J'ai été intentionnellement bousculé(e)

12. Une certaine force physique a été utilisée à mon encontre

13. J'ai reçu des propositions insistantes à caractère sexuel

14. Des tâches inférieures à mes compétences me sont systématiquement confiées

15. Des affiches à caractère pornographique ou dégradantes ont été placardées dans mon environnement de travail

16. J'ai subi des attouchements sans mon consentement 